\title{
Effect of End Group-Substrate Interaction on Aggregation Structure of Polystyrene Ultrathin Films
}

\author{
EL Sayed Mounir, Atsushi Takahara, and Tisato Kajiyama ${ }^{\dagger}$ \\ Department of Materials Physics and Chemistry, \\ Graduate School of Engineering, Kyushu University, \\ 6-10-1 Hakozaki, Higashi-ku, Fukuoka 812-8581, Japan
}

(Received August 24, 1998)

\begin{abstract}
The aggregation structure of proton-, monocarboxyl-, and dicarboxyl terminated polystyrene (PS) ultrathin films spin-coated onto different substrate surfaces was investigated on the basis of atomic force microscopy (AFM). In the case of the monocarboxyl- and dicarboxyl-terminated PS ultrathin films spin-coated onto Si-wafer, the dewetting occurred after annealing above their bulk glass transition temperatures. In contrast, those (monocarboxyl and dicarboxyl-terminated PS ultrathin films spin-coated) onto aminosilane-treated Si-wafer did not dewet even after annealing above the bulk $T_{\mathrm{g}}$, owing to the specific interaction between $-\mathrm{COOH}$ and $-\mathrm{NH}_{2}$ groups of aminosilane onto the Si-wafer.

KEY WORDS Polystyrene Ultrathin Films / Dewetting / End Group-Substrate Interaction / Atomic Force Microscopy / Monocarboxyl-Terminated Polystyrrene / Dicarboxyl-Terminated Polystyrene /
\end{abstract}

Ultrathin polymer films are becoming more interesting from technological application and scientific standpoints. Also, polymeric surface functions as biomaterials, adhesives, lubricants, and others, are closely related to the surface aggregation structure of polymeric solids. ${ }^{1,2}$ Therefore, studies on surface aggregation structure for polymeric thin films are important for practical applications as well as their scientific interest. Surface aggregation structure of polymer thin films has been investigated, both experimentally and theoretically. ${ }^{3-6}$ The stability of aggregation structure for the ultrathin films on solid substrate is of great importance in many industrial applications, and its controlling parameter might be wettability against substrate. ${ }^{7,8}$ However, it is often experienced that the polymer ultrathin films dewet the substrate upon heating above its glass transition temperature, $T_{\mathrm{g}}{ }^{9,10}$ The dewetting of aggregation structure of end-functionalized polymer ultrathin film has been reported. ${ }^{11-13}$ To achieve long-term stabilization of surface aggregation structure for the ultrathin films, one may use polymers which can be anchored by one end group or two end groups to the substrate via strong chemical interaction. Klein et al. ${ }^{14,15}$ have showed that the thin films of an oligostyrene liquid, which are forced on a nonwetted Si-wafer surface, rupture within a few minutes. These films may be stabilized and rupture-inhibited for many months by the presence of a polymer brush on the $\mathrm{Si}$-wafer surface, together with some free polymer. The presence of both the brush and a sufficient amount of the free long-chain polymer are necessary for the prevention of rupture. Then the stability of aggregation structure of the end-functionalized polymer ultrathin film must be studied from the viewpoint of the film thickness and the degree of interaction between end group and substrate.

In this study, a promising technical approach to avoid the dewetting of aggregation structure for polymer ultrathin films have been proposed via specific interaction between carboxylated polystyrene and aminosilane-

\footnotetext{
† To whom correspondence should be addressed.
}

treated Si-wafer. Also, the effects of polymer film thickness on the stability of aggregation structure of the ultrathin polymer films have been studied by using the monocarboxyl- and dicarboxyl-terminated polystyrenes (PSs) and proton-terminated polystyrene (PS-H) as a reference.

\section{EXPERIMENTAL}

\section{Materials}

Polymers used in this study were proton-terminated polystyrenes (PS-H. $M_{n}=19.7 \mathrm{k}, M_{w} / M_{n}=1.08$ ), monocarboxyl-terminated polystyrenes (PSCOOH. $M_{n}=9.7 \mathrm{k}$, $M_{w} / M_{n}=1.18$, Functionality $\left.=0.98\right)$ and dicarboxylterminated polystyrenes $\left(\alpha, \omega-\mathrm{PS}(\mathrm{COOH})_{2} . M_{n}=12.0 \mathrm{k}\right.$, $M_{w} / M_{n}=1.09$, Functionality $\left.=1.98\right)$. The PSCOOH, and the $\alpha, \omega$-PS $(\mathrm{COOH})_{2}$ were purchased from Polymer Source Inc., while monodisperse proton-terminated polystyrenes (PS-H) were synthesized by a living anionic polymerization with $s$-butyllithium as an anionic monofunctional initiator.

\section{Film Preparation}

The PS thin films were prepared by spin-coating at $2500 \mathrm{rpm}$ from a toluene solution of $0.1-0.05 \mathrm{wt} \%$ onto different substrate. Substrates used in this study were native oxide Si-wafer and aminosilanized Si-wafer. An aminosilane in a vapor state was applied in order to obtain homogeneous and flat monolayer. ${ }^{16}$ The aminosilanized Si-wafer was used to enhance the interaction between carboxyl-end group and substrate. Aminosilanization was carried out by chemisorption of 4aminobutyl-mono-methoxy-dimethylsilane (ABDMS) on clean and dried Si-wafer by following procedure. The substrate was placed on a teflon holder that was fixed in a round glass reactor, $0.2 \mathrm{ml}$ of ABDMS was dropped into a glass reactor by syringe. The glass reactor was evacuated at $20 \mathrm{mmHg}$ and then heated to $373 \mathrm{~K}$ for $2 \mathrm{~h}$. ABDMS was evaporated and silanization was carried out from a vapor phase. After silanization, the samples were rinsed with chloroform to remove unbound 
ABDMS.

\section{Characterization}

The bulk glass transition temperatures, $T_{\mathrm{g}}$ of the PS- $\mathrm{H}$, the PSCOOH, and the $\alpha, \omega$-PS $(\mathrm{COOH})_{2}$ were measured by differential scanning calorimetry (DSC 3200, MAC Science Co., Ltd.) under dried nitrogen atmosphere with heating rate of $10 \mathrm{~K} \mathrm{~min}^{-1}$.

FT-IR-external reflection spectroscopy (FT-IR-ERS) spectrum was obtained with a Nicolet, MAGNA-IR 860 spectrometer. The specimen for IR-ERS measurement was polymer film chemisorbed onto aminosilane treated $\mathrm{Si}$-wafer for $24 \mathrm{~h}$, and annealed at $393 \mathrm{~K}$ for $8 \mathrm{~h}$. The weakly adsorbed PS was extracted by cyclohexane. Then the IR spectrum of the PS strongly interacted with $\mathrm{NH}_{2}$ group was measured using nitrogen-cooled $\mathrm{HgCdTe}$ narrow-band detector, and grazing incidence $\left(80^{\circ}\right)$ reflection. The spectrum of the film was recorded by making 516 scans from $1800-1400 \mathrm{~cm}^{-1}$ with a resolution of $4 \mathrm{~cm}^{-1}$.

The film thickness was measured by optical ellipsometer (JASCO M-150) at incident angle of 55 degree with He-Ne laser $(\lambda=632.8 \mathrm{~nm})$.

The surface morphology of the PS-H, the PSCOOH, and the $\alpha, \omega$-PS $(\mathrm{COOH})_{2}$ ultrathin films spin-coated onto different substrates at different annealing treatment, were observed on the basis of atomic force microscopy (AFM). AFM images were obtained by SPA 300 with SPI 3700 controller (Seiko Instruments Industries Co., Ltd.) at room temperature. AFM cantilevers were microfabricated from $\mathrm{Si}_{3} \mathrm{~N}_{4}$ with spring constant of $0.02 \mathrm{~N} \mathrm{~m}^{-1}$.

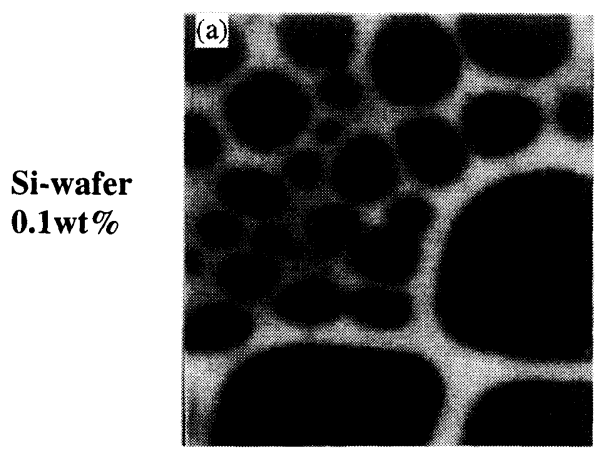

Step height ca. 40 nm

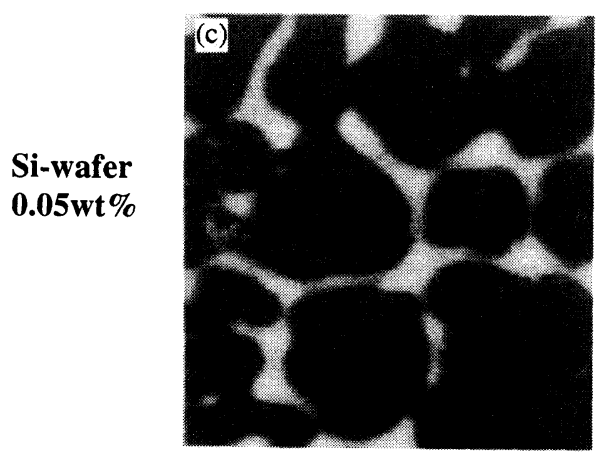

Step height ca. 32 nm

as cast
AFM imaging was carried out in a repulsive force of $0.022 \mathrm{nN}$ in air.

\section{RESULTS AND DISCUSSION}

\section{Bulk Characterization}

The physicochemical properties of polymers used in this study were summarized in Table I. The magnitude of $T_{\mathrm{g}}$ was in the order of PS-H $<$ PS-COOH $<\alpha, \omega$-PS$(\mathrm{COOH})_{2}$ even though these polymers had almost comparable $M_{n}$. The temperature at the midpoint of the base line shift was defined as $T_{\mathrm{g}}$ in this study. The DSC results indicate that $T_{\mathrm{g}} \mathrm{s}$ of the carboxyl terminated PSs were higher than that of the proton-terminated ones due to a decrease in chain end mobility and/or an increase in apparent molecular weight resulting from chain end association between carboxyl end groups.

\section{Effect of Polymer Solution Concentration on Aggregation Structure of PS Ultrathin Films}

The PS-H ultrathin films were prepared onto Si-wafer. The film thickness was decreased with a decrease in a

Table I. The physicochemical properties of proton terminated PS and carboxyl-terminated PSs

\begin{tabular}{lrccc} 
& $M_{n}$ & $M_{w} / M_{n}$ & $2 R_{\mathrm{g}} / \mathrm{nm}$ & $T_{\mathrm{g}} / \mathrm{K}$ \\
\hline PS-H & $19.7 \mathrm{k}$ & 1.08 & 7.6 & 364 \\
PSCOOH & $9.7 \mathrm{k}$ & 1.18 & 6.0 & 375 \\
$\alpha, \omega$-PS(COOH) & $12.0 \mathrm{k}$ & 1.08 & 6.1 & 383 \\
\hline
\end{tabular}

$R_{\mathrm{g}}$, radius of gyration

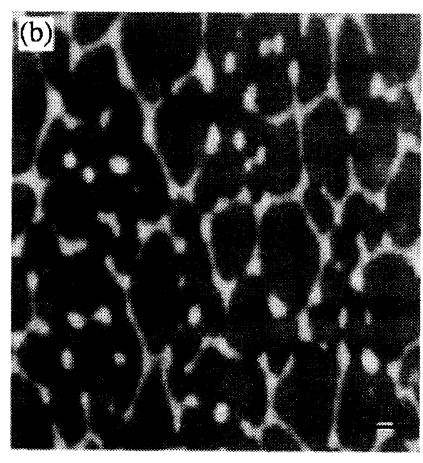

Step height ca. $25 \mathrm{~nm}$

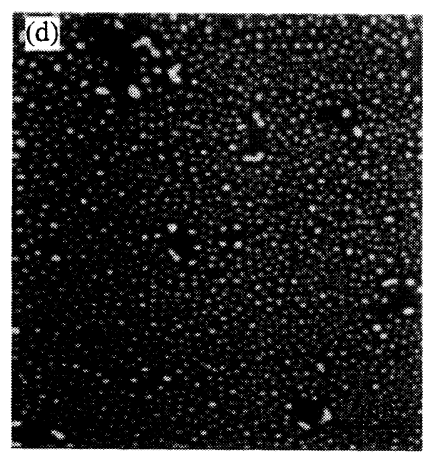

Step height ca. $18 \mathrm{~nm}$

annealed at $393 \mathrm{~K}$

for $8 \mathrm{~h}$

Figure 1. AFM images of proton-terminated PSs spin-coated onto Si-wafer from 0.1 and $0.05 w t \%$ of polymer solution before and after annealing. 


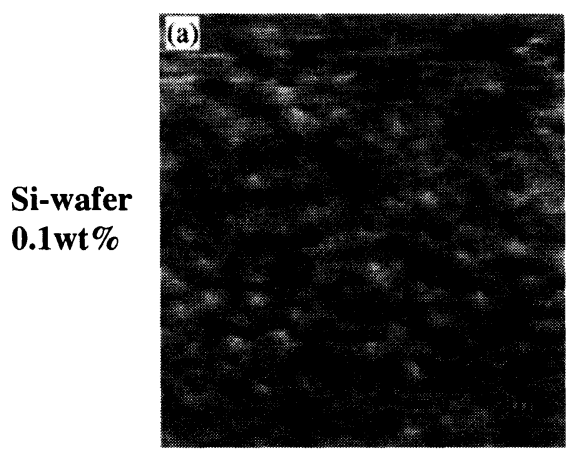

Thickness 9 nm

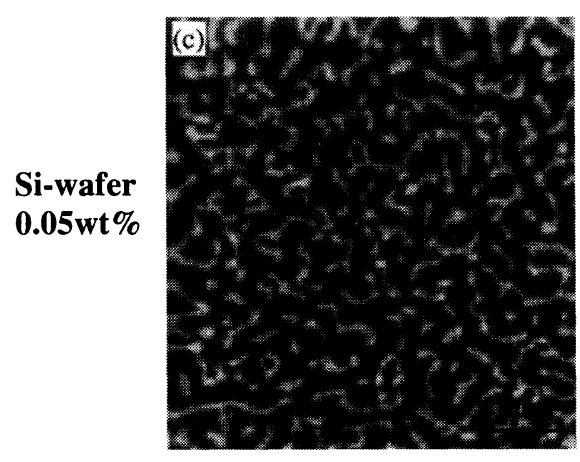

Step height ca. $25 \mathrm{~nm}$

(Mn 9.7k)

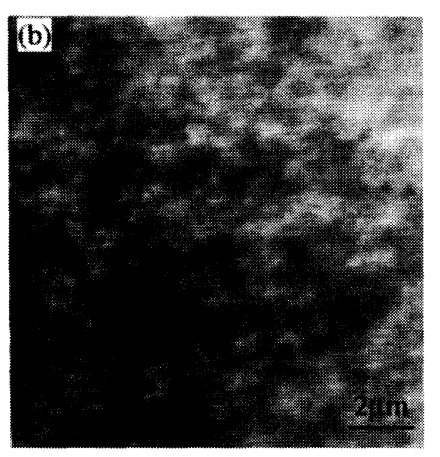

Thickness $10 \mathrm{~nm}$

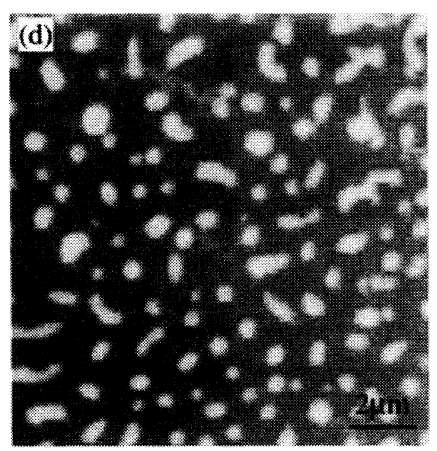

Step height ca. $22 \mathrm{~nm}$

(Mn 12.0k)

$\alpha, \omega-\mathrm{PS}(\mathbf{C O O H})_{2}$

Figure 2. AFM images of monocarboxyl- and dicarboxyl-terminated PSs spin-coated onto Si-wafer from $0.1 \mathrm{wt} \%$ [(a), (b)] and $0.05 \mathrm{wt} \%$ $[(\mathrm{c}),(\mathrm{d})]$ of polymer solution before annealing.

polymer solution concentration. Figure 1 shows AFM images of PSs spin-coated onto Si-wafer from toluene solutions of $0.1 \mathrm{wt} \%$ and $0.05 \mathrm{wt} \%$. The AFM images were obtained before and after annealing at $393 \mathrm{~K}$ for $8 \mathrm{~h}$. The bright part corresponds to PS phase, while the darker one to bare Si-wafer. In the case of the $0.1 \mathrm{wt} \%$ PS-H solution, the height of PS phase was $c a .40 \mathrm{~nm}$ before annealing as shown in Figure 1(a). When the annealing treatment was carried out at $393 \mathrm{~K}$ above its bulk $T_{\mathrm{g}}$, the height decreased to be $c a .25 \mathrm{~nm}$ as shown in Figure 1(b). Also, the PS height of dewetting structure prepared from the $0.05 \mathrm{wt} \%$ toluene solution was $c a$. $32 \mathrm{~nm}$ before annealing and decreased to $c a .18 \mathrm{~nm}$ after annealing treatment as shown in Figures 1(c) and (d). The AFM images revealed that the PS dewetted on the Siwafer with network-like structure even before annealing treatment. It might be reasonable to conclude that the absence of strong interaction between chain end groups and substrate cannot prevent dewetting of PS chains even before an annealing treatment. Also, it should be noted that the molecular weight of PS-H is below critical entanglement molecular weight, $M_{e} \cdot{ }^{17}$ The continuity of dewetted phase decrease after annealing treatment, especially in Figures 1(c) and (d). The dewetting behavior became more apparent with a decrease in the solution concentration, and also annealing treatment above bulk $T_{\mathrm{g}}$. The dewetting of PS-H on Si-wafer upon annealing might be attributed due to the absence of strong interaction between PS-H chains and substrate.
Effect of End Group-Substrate Interaction on the Dewetting Behavior of PS Ultrathin Films

In order to enhance an interaction between polymer chain ends and substrate, PSs with monocarboxyl-end group and dicarboxyl-one were employed here. Figure 2 shows the AFM images of monocarboxyl- and dicarboxyl-terminated PS ultrathin films spin-coated onto Si-wafer prepared from the $0.1 \mathrm{wt} \%$ and $0.05 \mathrm{wt} \%$ polymer solutions. The ultrathin films prepared from the $0.1 \mathrm{wt} \%$ solution were homogeneous, being independent of the functionality of polymer chain as shown in Figures 2(a) and (b). The film thickness was ca. $10 \mathrm{~nm}$ being larger than $2 R_{\mathrm{g}}$. On the other hand, the film prepared from the $0.05 \mathrm{wt} \%$ polymer solution showed dewetting structure with the height of $c a .22-25 \mathrm{~nm}$ as shown in Figures 2(c) and (d), since the brighter and darker regions correspond to the PS phase and the bare substrate, respectively. The less extensive dewetting behavior of the PSCOOH and the $\alpha, \omega$-PS $(\mathrm{COOH})_{2}$ on Si-wafer in comparison with that of PS-H can be attributed to the hydrogen bonding interaction between $-\mathrm{COOH}$ chain ends and $\mathrm{OH}$ on oxidized Si-wafer.

The aminosilane-treated $\mathrm{Si}$-wafer was used as the substrate in order to improve the substrate-polymer interaction. Figure 3 shows the AFM images of as-spincoated monocarboxyl- and dicarboxyl-terminated PS ultrathin films onto aminosilane-treated Si-wafer from the $0.1 \mathrm{wt} \%$ and $0.05 \mathrm{wt} \%$ polymer solutions. In the case of the PSCOOH and the $\alpha, \omega$-PS $(\mathrm{COOH})_{2}$, the uniform thin film could be formed on the aminosilane- 


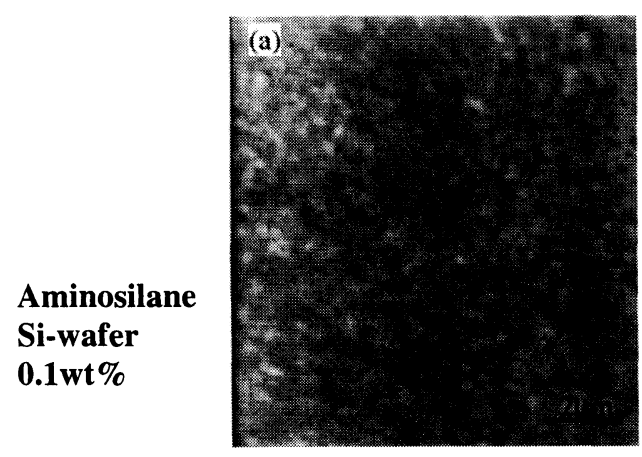

Thickness $12 \mathrm{~nm}$

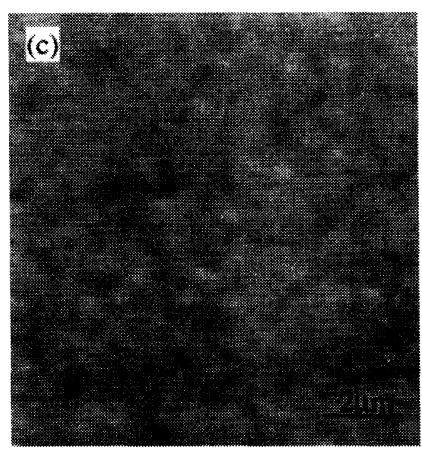

Thickness 8 nm

(Mn 9.7k)

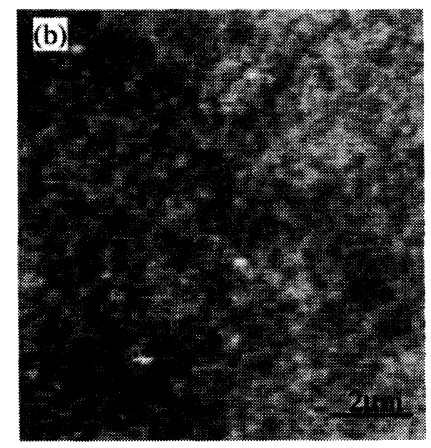

Thickness $13 \mathrm{~nm}$

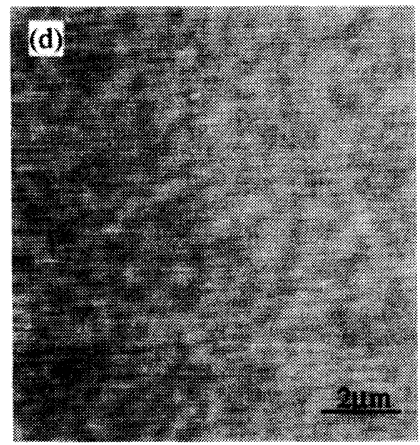

Thickness 9 nm

(Mn12.0k)

$\alpha, \omega-\mathrm{PS}(\mathbf{C O O H})_{2}$

Figure 3. AFM images of monocarboxyl- and dicarboxyl-terminated PSs spin-coated onto aminosilane-treated Si-wafer from $0.1 \mathrm{wt} \%$ [(a), (b)] and $0.05 \mathrm{wt} \%[(\mathrm{c}),(\mathrm{d})]$ of polymer solution before annealing.

treated Si-wafer before annealing, being independent on the concentration of the polymer solution as shown in Figures 3(a)-(d). The film thickness prepared from 0.1 and $0.05 \mathrm{wt} \%$ polymer solutions were $c a .12 .5$ and $8.5 \mathrm{~nm}$, respectively. These thicknesses were larger than $2 R_{\mathrm{g}}$ of an unperturbed chain.

Since an anchoring of chain end group to the substrate would influence the polymer chain mobility, the annealing effect on the molecular aggregation state for PSCOOH and $\alpha, \omega$-PS $(\mathrm{COOH})_{2}$ films above its bulk $T_{\mathrm{g}}$ was investigated. Figure 4 shows the AFM images of the $\mathrm{PSCOOH}$ and the $\alpha, \omega$-PS $(\mathrm{COOH})_{2}$ films spin-coated from the $0.1 \mathrm{wt} \%$ solution onto $\mathrm{Si}$-wafer and aminosilane-treated Si-wafer substrates and annealed at $393 \mathrm{~K}$ for $8 \mathrm{~h}$. When $\mathrm{Si}$-wafer was used as the substrate, the PSCOOH thin film showed dewetting structure with droplet-like PS phase of $27-30 \mathrm{~nm}$ high as shown in Figure 4(a), while the $\alpha, \omega-\mathrm{PS}(\mathrm{COOH})_{2}$ showed partial dewetting structure with holes of $10-12 \mathrm{~nm}$ in depth as shown in Figure 4(b). The brighter and darker parts correspond to PS phase and bare substrate, respectively. A slightly improved wetting property for the $\alpha, \omega$ $\mathrm{PS}(\mathrm{COOH})_{2}$ thin film on Si-wafer in comparison with the PSCOOH film may be interpreted in terms of the number density of hydrogen bonding between $-\mathrm{COOH}$ chain ends and HO-Si on the substrate. On the other hand, in the case of aminosilane-treated Si-wafer substrate, both the PSCOOH and the $\alpha, \omega-\mathrm{PS}(\mathrm{COOH})_{2}$ formed the uniform thin films with $11-12 \mathrm{~nm}$ thick even after annealing treatment as shown in Figures 4(c) and (d). The formation of the uniform thin films on aminosilane-treated Si-wafer after annealing might be attributed to stronger interaction between polymer chain end groups and substrate.

Figures 5 shows the AFM images of monocarboxyland dicarboxyl-terminated PSs spin-coated onto Si-wafer and onto aminosilane-treated Si-wafer from the $0.05 \mathrm{wt} \%$ polymer solution and annealed at $393 \mathrm{~K}$ for $8 \mathrm{~h}$. When Si-wafer was used as the substrate, the PSCOOH thin film showed apparent dewetting structure as shown in Figure 5(a). Upon annealing above its bulk $T_{\mathrm{g}}$, the interfacial interaction between the $-\mathrm{COOH}$ ends and $\mathrm{Si}$-wafer linkage was weakened, resulting in the formation of dewetting structure due to recovery of conformational entropy of polymer chains and in other word, their conformational rearrangement. The dewetting behavior became more prominent with a decrease in the film thickness as shown in Figure 5(a). The PSCOOH chains constrained in an extremely narrow space tend to recover its conformational entropy upon annealing at $393 \mathrm{~K}$ for $8 \mathrm{~h}$. The dewetting behavior of ultrathin film prepared from the $0.05 \mathrm{wt} \%$ solution was more enhanced than those prepared from the $0.1 \mathrm{wt} \%$ solution as shown in Figures 4 and 5 . In the case of the $\alpha, \omega-\mathrm{PS}(\mathrm{COOH})_{2}$, since the hydrogen bonding association among carboxyl groups is responsible for ionic association, the dewetting 


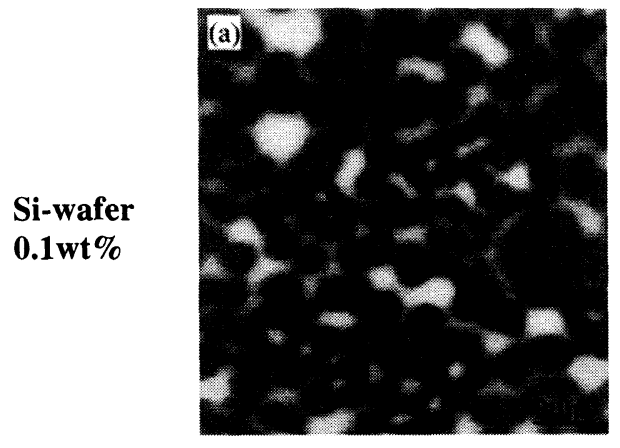

Step height ca. $27-30 \mathrm{~nm}$

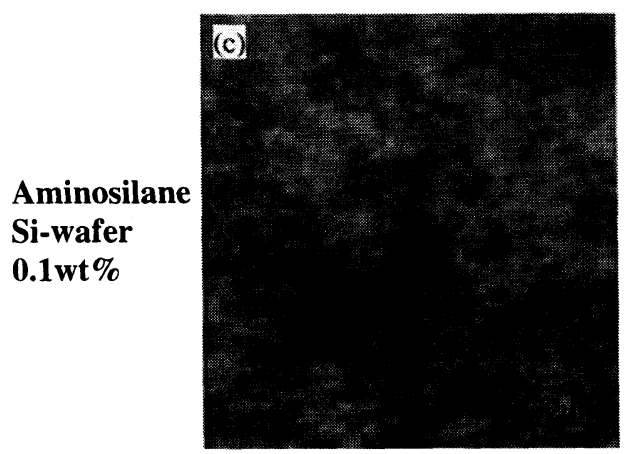

Thickness 11 nm

(Mn 9.7k)

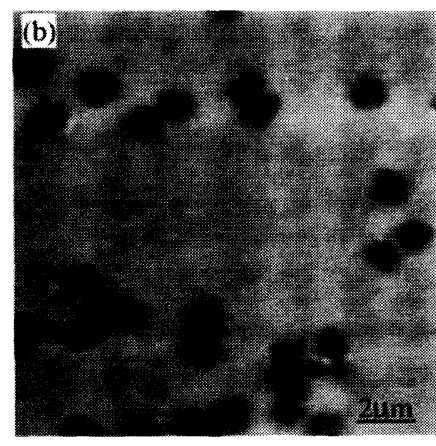

Step height ca. 10-12 nm

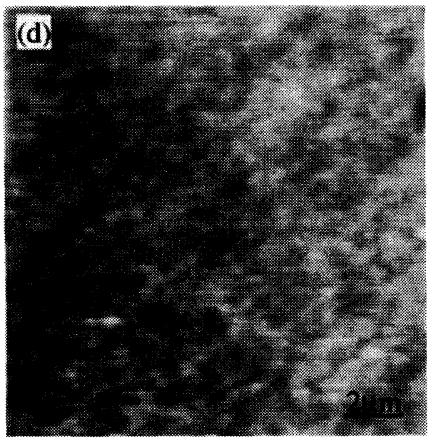

Thickness 12 nm

(Mn 12.0k)

\section{PSCOOH}

\section{$\alpha, \omega-\mathrm{PS}(\mathbf{C O O H})_{2}$}

Figure 4. AFM images of monocarboxyl- and dicarboxyl-terminated PSs spin-coated onto Si-wafer [(a), (b)], and onto aminosilane-treated Si-wafer $[(\mathrm{c}),(\mathrm{d})]$ from $0.1 \mathrm{wt} \%$ of polymer solution and annealed at $393 \mathrm{~K}$ for $8 \mathrm{~h}$.

occurred on Si-wafer upon annealing as shown in Figure 5 (b) due to reduction of ionic association between COOH groups and $\mathrm{HO}-\mathrm{Si}$ on $\mathrm{Si}$-wafer substrate. ${ }^{14} \mathrm{On}$ the other hand, in the case of the aminosilane-treated Si-wafer substrate, the dewetting structures was not observed as shown in Figures 5(c) and (d), may be due to the formation of specific interaction between $-\mathrm{COOH}$ and $\mathrm{NH}_{2}$ groups of aminosilane on Si-wafer. The $\mathrm{NH}_{2}$ groups of ABDMS might form stronger interaction with PS carboxyl chain end groups, in comparison with the hydrogen bonding association that is easily dissociated upon an annealing treatment. The formation of amide between $-\mathrm{COOH}$ and $-\mathrm{NH}_{2}$ make the polymer ultrathin films wetted uniformly and homogeneously on the aminosilane treated Si-wafer.

In order to investigate the degree of interaction between $-\mathrm{NH}_{2}$ and $-\mathrm{COOH}$ groups, FT-IR reflectance spectrum was obtained. Figure 6 shows the FT-IRexternal reflection spectrum to confirm a certain kind of chemical interaction between PSCOOH and amine group of aminosilane-treated Si-wafer. The sample was annealed at $393 \mathrm{~K}$ for $8 \mathrm{~h}$, and then, the weakly adsorbed PS to the substrate was extracted with cyclohexane. Therefore, only the PS strongly interacted with the $\mathrm{NH}_{2}$ group was present on the substrate surface. The strong absorption at $1600 \mathrm{~cm}^{-1}$ was assigned to the $\mathrm{C}=\mathrm{C}$ stretching of aromatic ring of PS. The apparent absorption peaks of both stretching vibration of $(-\mathrm{CONH}-)$ amide linkage at $1670 \mathrm{~cm}^{-1}$ and hydrogen-bonded carbonyl at $1705 \mathrm{~cm}^{-1}$ clearly indicate strong chemical bond formation between substrate surface and polymer chain ends. Then, it is reasonable to consider that the strong chemical interaction between substrate and polymer chains effectively reduce dewetting due to a decrease in polymer chain diffusion, resulting in the formation of the homogeneous ultrathin films.

Figure 7 shows schematic representation of the possible state of chemical interaction and partially hydrogen bonding between carboxyl-end group and amine group of aminosilane-treated Si-wafer. The $\mathrm{NH}_{2}$ groups of ABDMS form amide linkage with PS chain end groups, rather than the formation of hydrogen bonding association that is easily dissociated upon annealing treatment. The chemical interaction between $-\mathrm{COOH}$ and $-\mathrm{NH}_{2}$ may make the polymer ultrathin films wetted uniformly and homogeneously on the aminosilanetreated Si-wafer.

\section{CONCLUSION}

Wetting behavior of ultrathin films of end-functionalized monocarboxyl- and dicarboxyl-terminated PSs spin-coated onto $\mathrm{Si}$-wafer and aminosilane-treated $\mathrm{Si}$ - substrate from the $0.1 \mathrm{wt} \%$ and the $0.05 \mathrm{wt} \%$ solution, has been discussed as a function of film thickness (solution concentration), and annealing treatment. The polymer ultrathin films of monocarboxyl-terminated PS did not form homogeneous and stable structure on the Si-wafer substrate. This might be attributed to conformational rearrangement of a $\mathrm{PSCOOH}$ in narrow 


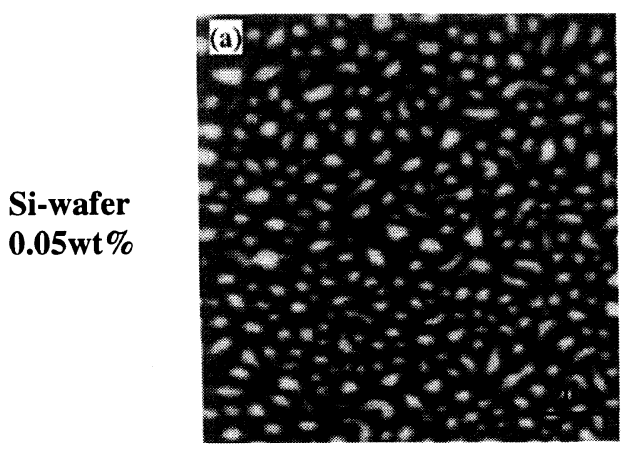

Step height ca. $32 \mathrm{~nm}$

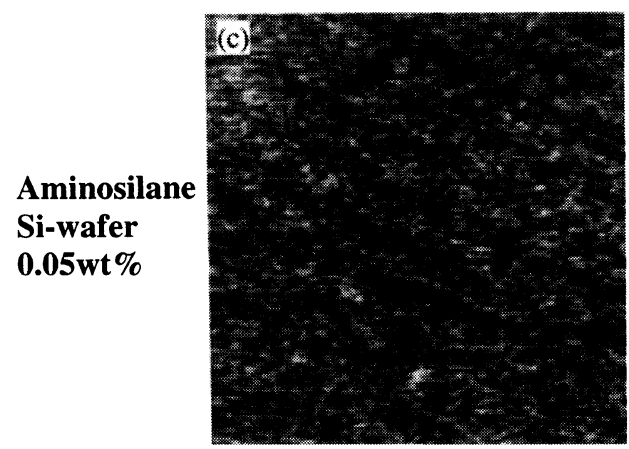

Thickness 9 nm

(Mn 9.7k)

PSCOOH

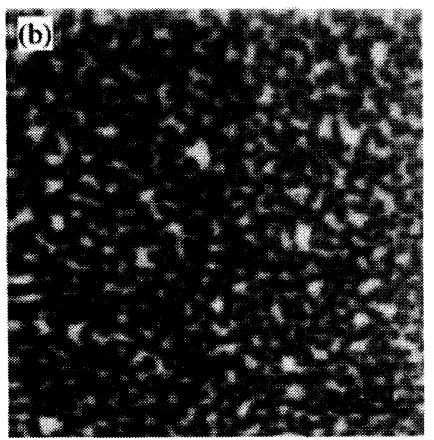

Step height ca. $29 \mathrm{~nm}$

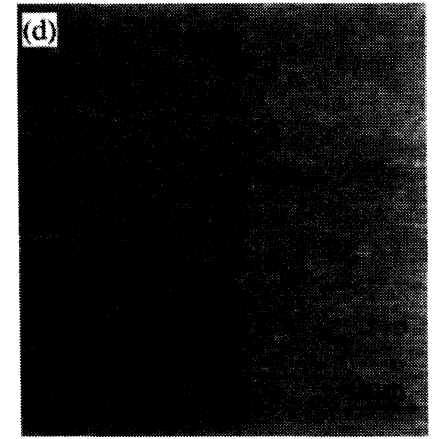

Thickness 9 nm

(Mn 12.0k)

$\alpha, \omega-\mathrm{PS}(\mathbf{C O O H})_{2}$

Figure 5. AFM images of monocarboxyl- and dicarboxyl-terminated PSs spin-coated onto Si-wafer [(a), (b)], and onto aminosilane-treated Si-wafer [(c), (d)] from $0.05 \mathrm{wt} \%$ of polymer solution and annealed at $393 \mathrm{~K}$ for $8 \mathrm{~h}$.

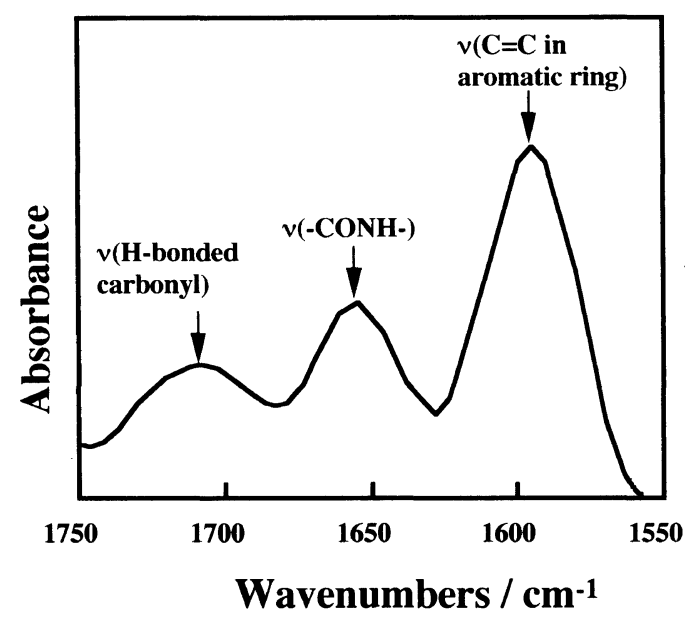

Figure 6. FT-IR-ERS spectrum of carboxyl-terminated PS prepared onto aminosilane-treated $\mathrm{Si}$-wafer.

space to recover its conformational entropy due to annealing treatment above bulk $T_{\mathrm{g}}$. While, in the case of Si-wafer, the $\alpha, \omega$-PS $(\mathrm{COOH})_{2}$ ultrathin film showed a little improvement in wetting properties in comparison with that of the PSCOOH film. Since the interaction between $-\mathrm{COOH}$ and Si-wafer linkage was weakened upon annealing above its bulk glass transition temperature, annealing treatment induced conformational rearrangement of a polymer chain and then, dewetting of PSCOOH and $\alpha, \omega$-PS(COOH $)_{2}$ were easily occurred. On the other hand, the dewetting of monocarboxyl- and

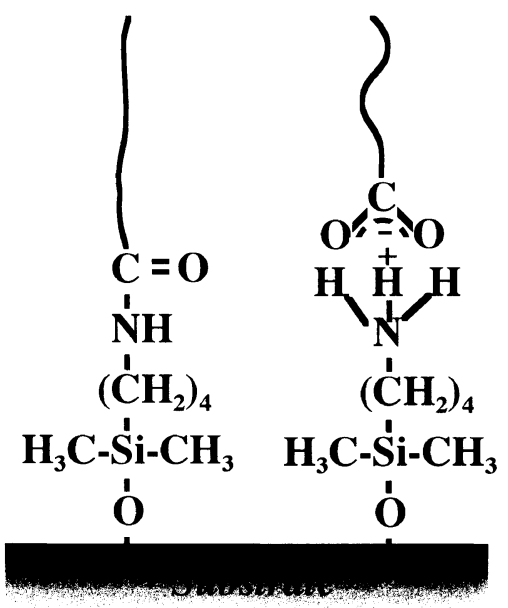

Figure 7. Schematic representation of the possible state of interaction between carboxyl-end group and aminosilane-treated Si-wafer.

dicarboxyl-terminated PSs onto aminosilane-treated Si-wafer prevented effectively, regardless of both polymer film thickness and annealing treatment. Improved wetting behavior of the PSCOOH and the $\alpha, \omega$ $\mathrm{PS}(\mathrm{COOH})_{2}$ may be attributed to the formation of amide linkage between $-\mathrm{COOH}$ of $\mathrm{PS}$ and $\mathrm{NH}_{2}$ group of aminosilane-treated Si-wafer. The results mentioned above clearly indicate that substrate-polymer chain end interaction plays an important role in the mobility of polymer chains and dewetting characteristics on the 
substrate.

\section{REFERENCES}

1. W. J. Feast, H. S. Munro, and R.W. Richards, " Polymer Surface and Interfaces," Vol. 2, Wiley, New York, N.Y., 1993.

2. F. Garbassi, M. Morra, and E. Occhiello "Polymer Surfaces from Physics to Technology," Wiley, New York, N.Y., 1994.

3. H. Nakanishi and P. Pincus J. Chem. Phys., 79, 997 (1983).

4. R. A. L. Jones and E. J. Kramer, Polymer, 34, 115 (1993).

5. K. Tanaka, A. Takahara, and T. Kajiyama, Macromolecules, 29 , $3232(1996)$

6. T. Kajiyama, K. Tanaka, S.-R. Ge, and A. Takahara, Prog. Surf. Sci., 52, 1 (1996).

7. A. Sharma, Langmuir, 9, 861 (1993).
8. I. Langmuir, Trans. Faraday Soc., 15, 62 (1920).

9. D. J. Srolowitz and S. A. Safran, J. Appl. Phys., 60, 247 (1986).

10. R. Sekimoto, K. Oguma, and A. Kawasaki, Ann. Phys. (N.Y.), 176, 35 (1987).

11. G. Reiter, P. Auroy, and L. Auvray, Macromolecules, 29, 2150 (1996).

12. W. Mormann and K. Schmalz, Macromolecules, 27, 7115(1994).

13. L. J. Fetters, D. J. Lohse, D. Richter, T. A. Witten, and A. Zirke, Macromolecules, 27, 4639 (1994).

14. R. Y. Rozen and J. Klein, Langmuir, 11, 2806 (1995)

15. R. Y. Rozen, J. Klein, and L. J. Fetters, Science, 263, 793 (1994).

16. D. Bayer, T. M. Bohanon, W. Knoll, H. Ringsdorf, G. Elender, and E. Sackmann, Langmuir, 12, 2514 (1996).

17. W. W. Graessley, "Physical Properties of Polymers," American Chemical Society, New York, N.Y., 1984, Chapter III. 